\title{
Orbital-selective superconductivity in a two-band model of infinite-layer nickelates
}

\author{
Priyo Adhikary $\odot,{ }^{1, *}$ Subhadeep Bandyopadhyay, ${ }^{2, *}$ Tanmoy Das $\odot,{ }^{1, \dagger}$ Indra Dasgupta, ${ }^{2, \ddagger}$ and Tanusri Saha-Dasgupta $\odot^{3, \S}$ \\ ${ }^{1}$ Department of Physics, Indian Institute of Science, Bangalore 560012, India \\ ${ }^{2}$ School of Physical Sciences, Indian Association for the Cultivation of Science, Kolkata 700032 , India \\ ${ }^{3}$ S. N. Bose National Centre for Basic Sciences, JD Block, Sector III, Salt Lake, Kolkata, West Bengal 700106, India
}

(Received 3 May 2020; revised 18 July 2020; accepted 18 August 2020; published 1 September 2020)

\begin{abstract}
In the present Rapid Communication, we explore superconductivity in $\mathrm{NdNiO}_{2}$ and $\mathrm{LaNiO}_{2}$ employing a first-principles derived low-energy model Hamiltonian, consisting of two orbitals: Ni $x^{2}-y^{2}$, and an axial orbital. The axial orbital is constructed out of $\mathrm{Nd} / \mathrm{La} d, \mathrm{Ni} 3 z^{2}-r^{2}$, and Ni $s$ characters. Calculation of the superconducting pairing symmetry and pairing eigenvalue of the spin-fluctuation mediated pairing interaction underlines the crucial role of the interorbital Hubbard interaction in superconductivity, which turns out to be orbital selective. The axial orbital brings in material dependence to the problem, making $\mathrm{NdNiO}_{2}$ different from $\mathrm{LaNiO}_{2}$, thereby controlling the interorbital Hubbard interaction-assisted superconductivity.
\end{abstract}

DOI: 10.1103/PhysRevB.102.100501

Introduction. While the mechanism of superconductivity in cuprates [1] still remains debated, attempts have been made in the search for superconductivity in transition-metal oxides other than cuprates. Nickelates, $\mathrm{Ni}$ being the neighboring element to $\mathrm{Cu}$, turns out to be most promising in this respect. Towards this goal, a number of attempts has been made [2-7], but superconductivity in these systems remained elusive. In this connection, the stabilization of unusual $3 d^{9}, \mathrm{Ni}^{1+}$ valence in $\mathrm{LaNiO}_{2}$ and $\mathrm{NdNiO}_{2}[8,9]$ has generated interest. A recent report [10] of superconductivity in $20 \% \mathrm{Sr}$-doped $\mathrm{NdNiO}_{2}$ with a $T_{c}$ of $9-15 \mathrm{~K}$ has reignited this interest with reports of several theoretical studies [11-23] devoted to its understanding.

The most sought after issue, in this respect, has been whether $\mathrm{Nd} / \mathrm{LaNiO}_{2}(\mathrm{~N} / \mathrm{LNO})$ can be described within the same theoretical framework as cuprates. While undoped cuprates are strong insulators [24], undoped N/LNO is reported to be a bad metal $[8,10]$. Comparing the electronic structure with that of cuprates, the most obvious difference is the large $\mathrm{O} 2 p-\mathrm{Ni} 3 d$ charge transfer energy for $\mathrm{Ni}^{1+}$ compared to the small $\mathrm{O} 2 p-\mathrm{Cu} 3 d$ charge transfer energy for $\mathrm{Cu}^{2+}$, resulting in a significantly weaker hybridization with O $2 p$ compared to cuprates. This fact has been evidenced in electronic structure calculations $[13,18]$ as well as in terms of the absence of a prepeak in X-ray absorption spectroscopy (XAS) near the $\mathrm{O} K$ edge [25]. This also provides justification for the weak superexchange in NNO compared to strong antiferromagnetism in undoped cuprates [26]. The large charge transfer energy puts the $\mathrm{Ni} 3 d$ levels higher up in energy compared to $\mathrm{Cu} 3 d$, which facilitates their hybridization with usually empty $\mathrm{Nd} / \mathrm{La} d$ bands. While the hybridization of in-plane $\mathrm{Ni} x^{2}-y^{2}$ with out-of-plane $\mathrm{Nd} / \mathrm{La} d$ orbitals is

\footnotetext{
${ }^{*}$ These authors contributed equally to this work.

†tnmydas@gmail.com

${ }^{\ddagger}$ sspid@iacs.res.in

\$t.sahadasgupta@gmail.com
}

negligible, they can hybridize via Ni $3 z^{2}-r^{2}$ and Ni $s[18,27]$. The Nd/La-Ni hybridization has been signaled in additional low-energy shoulder features in XAS and resonant inelastic Xray scattering (RIXS) spectra of N/LNO [25], with predicted mixing from rare earths as large as $44 \%$ [25]. Thus while in the case of cuprates, the intercalated non-copper-oxide layers act as spectators, playing the role of a simple "charge reservoir," in the case of N/LNO the rare-earth layer may provide active electronic degrees of freedom. This prompted Hepting et al. [25] to suggest a two-band model, consisting of a three-dimensional (3D) rare-earth band coupled to a $\mathrm{Ni}$ $x^{2}-y^{2}$ derived 2D Mott system. Interestingly, a more recent report shows a sign reversal of Hall resistivity with doping and temperature [28], which may further evince the two-band scenario.

Given this background, a natural question would be, can doped carriers give rise to superconductivity in such a twoband model description of NNO and LNO, and if so, is there any difference between NNO and LNO. This still needs to be explored, though superconductivity in nickelates has been explored within the framework of one-band and three-band models with on-site correlations [12], a one-band Hubbard model [14], a multiband $\mathrm{Ni} d-\mathrm{Nd} d$ Hubbard model within the fluctuation-exchange approximation [11], as well as from strong-coupling starting points [20-22].

In the present Rapid Communication, we first construct a two-band model, starting from the self-consistent-field density functional theory (DFT) and by retaining $\mathrm{Ni} x^{2}-y^{2}$ and $\mathrm{Ni} s$ orbitals in the basis, and downfolding the rest. Here, our main finding is that the $\mathrm{Ni} s$ basis forms an axial orbital, resulting from the hybridization of $\mathrm{Nd} / \mathrm{La} 3 z^{2}-r^{2}, \mathrm{Nd} / \mathrm{La}$ $x y, \mathrm{Ni} 3 z^{2}-r^{2}$, and $\mathrm{Ni} s$. Moreover, while the downfolded $\mathrm{Ni} x^{2}-y^{2}$ Wannier orbital is very similar in the La and $\mathrm{Nd}$ compounds, the detailed nature of the axial orbital sets these two materials apart, giving a clue as to its possible role in material-dependent superconductivity.

We next solve the pairing eigenvalue and pairing eigenfunctions of the spin-fluctuation mediated pairing 
interaction, computed within the DFT-derived two-Wannierorbital Hubbard model. We find that (i) in the Nd compound, the superconducting (SC) coupling constant $\lambda$ grows almost exponentially with the interorbital interaction $V_{s d}$, while the intraorbital interactions alone are not conducive for superconductivity. In a crude analogy with the renormalization theory, we can say that intraorbital interactions are "marginal"they do not directly mediate superconductivity-while the interorbital interaction is a "relevant" parameter for superconductivity. (ii) Second, in NNO, we find that the pairing eigenfunction turns out to be orbital selective: being 2D $x^{2}-y^{2}$ type for the Ni $d$ orbital, and 3D $3 z^{2}-r^{2}$-type symmetry for the axial orbital. The same study in LNO results in a single $x^{2}-y^{2}$ wave pairing symmetry, but with a SC coupling constant significantly smaller than that of NNO. Our findings emphasize the importance of the axial orbital and a two-band model in which the orbital-selective pairing symmetry is augmented by the interorbital interaction.

DFT band structure and two-band model. The DFT band structure is computed in the plane-wave basis, as implemented in the Vienna ab initio simulation package (VASP) [29] with the projected augmented-wave (PAW) potential [30] and choice of generalized gradient approximation [31] (GGA) for the exchange-correlation functional [see Supplemental Material (SM)] [32]. The results for undoped N/LNO are shown in Figs. 1(a) and 1(b). The band structure of N/LNO, which is well studied in literature $[12,17,18,33]$, primarily consists of $\mathrm{O}-2 p$ dominated bands ranging from about $-8 \mathrm{eV}$ to about $-3 \mathrm{eV}, \mathrm{Ni}-3 d$ dominated bands ranging from about -3 to $2 \mathrm{eV}$, and $\mathrm{Nd} / \mathrm{La}-5 d$ dominated bands ranging from about -1 to $8 \mathrm{eV}$. The low-energy electronic structure has two bands crossing the Fermi level: one canonical Ni $x^{2}-y^{2}$ band creating a hole pocket centered around the $M(A)$ point, bearing a strong resemblance to cuprates, and the other one is derived out of $\mathrm{Nd} / \mathrm{La} d$ mixed with $\mathrm{Ni}$ characters creating electron pockets at the $\Gamma$ and $A$ points. While the generic features are found to be similar in the band structures of NNO [Fig. 1(a)] and LNO [Fig. 1(b)], there are subtle differences. Comparing the Ni $x^{2}-y^{2}$ bands in the two compounds, the bandwidth of Ni $x^{2}-y^{2}$ in the LNO band is smaller by about $0.2 \mathrm{eV}$ as compared to NNO. The corresponding $k_{z}$ dispersion is also smaller for LNO compared to NNO. This $k_{z}$ dispersion highlights the mixing with the axial orbital, making the $\mathrm{Ni}$ $x^{2}-y^{2}$ band deviate from its $2 \mathrm{D}$ nature, as emphasized by Lee and Pickett [18]. Comparing the second band, we find that first the $\mathrm{Nd} d$-Ni derived electron pocket centered around $\Gamma$ is about $-0.4 \mathrm{eV}$ lower in energy in NNO as compared to LNO, making the self-doping effect more pronounced in the $\mathrm{Nd}$ compound compared to the La compound. Second, the width of the second band is about $1 \mathrm{eV}$ smaller in LNO compared to NNO.

These subtle but important material-specific differences of the electronic structure of nickelate compounds get reflected in the Wannier functions [34] defining the downfolded twoband structure, designed to reproduce the two low-energy bands of the DFT band structure. In order to construct the low-energy two-band structure, we retain $\mathrm{Ni} x^{2}-y^{2}$ and $\mathrm{Ni}$ $s$ degrees of freedom, and downfold the rest. This choice is guided by the four-band model of cuprates [35,36], with a material-dependent axial orbital formed by combining
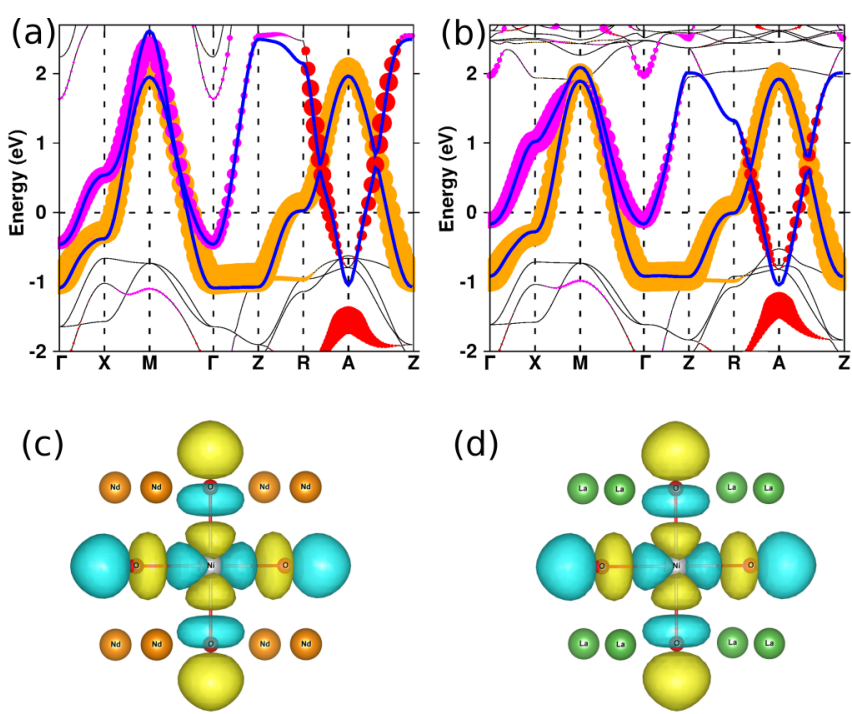

(d)
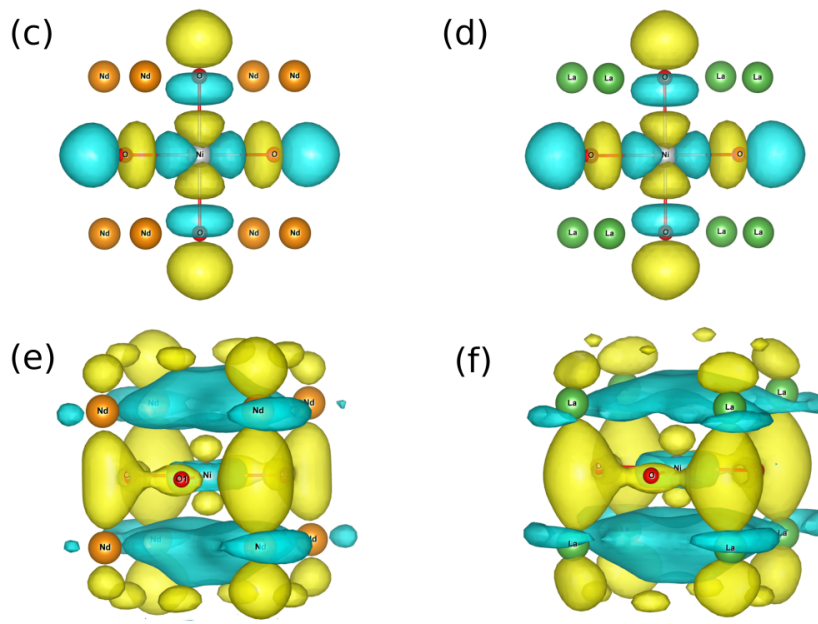

(f)

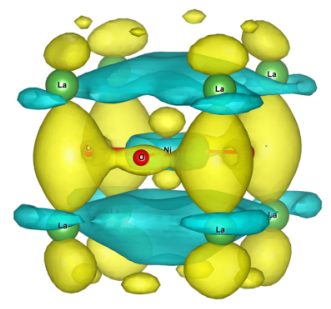

FIG. 1. (a), (b) The DFT band structure (thin, black) together with a downfolded two-band structure (thick, blue) for NNO and LNO, plotted along the high-symmetry points of a tetragonal unit cell, $\Gamma(0,0,0)-X(\pi / a, 0,0)-M(\pi / a, \pi / a, 0)-\Gamma-Z(0,0, \pi / c)-R(\pi / a, 0$, $\pi / c)-A(\pi / a, \pi / a, \pi / c)-Z$. The fatness in the DFT band structure corresponds to Ni $x^{2}-y^{2}$ (orange), $\mathrm{Nd} 3 z^{2}-r^{2}$ (magenta), and $\mathrm{Nd} x y$ (red). (c), (d) The Ni $x^{2}-y^{2}$ Wannier function in the downfolded two-band basis for NNO and LNO. (e), (f) Same as (c) and (d), but plotted for an axial orbital. Plotted are the constant value surfaces with lobes of different signs colored as yellow and cyan.

$\mathrm{Cu} s, \mathrm{Cu} 3 z^{2}-r^{2}$, apical oxygen $p_{z}$, and orbitals of farther axial cations. In the present case, starting from such a four-band model, we further downfold $\mathrm{O} p_{x}$ and $\mathrm{O} p_{y}$ due to the larger charge transfer energy between $\mathrm{Nd} d$ and $\mathrm{O} p$ in nickelates, compared to cuprates. We find that matching between the downfolded two-band and DFT bands (see Fig. 1 and SM) is as good as that obtained within the three-band model in Ref. [17] and the 17 Wannier projection bands in Ref. [16], justifying the goodness and sufficiency of the minimal twoorbital model.

The resultant Wannier functions corresponding to the downfolded two-band structure are shown in Figs. 1(c)-1(f) for N/LNO. The $x^{2}-y^{2}$ Wannier function which forms the $p d \sigma$ antibonding combination [Figs. 1(c)and 1(d)] is found to be identical between the $\mathrm{Nd}$ and $\mathrm{La}$ compounds. We note that the $p$-like tail of the $x^{2}-y^{2}$ Wannier functions sitting at the $\mathrm{O}$ sites shows asymmetry between the positive and negative lobes, which signifies the mixing with diffuse $\mathrm{Ni} s$. The material dependence, however, shows up in the Wannier function which forms the axial orbital [Figs. 1(e) and 1(f)]. We find this axial orbital is a hybrid between Ni $s$, 
$\mathrm{Ni} 3 z^{2}-r^{2}, \mathrm{Nd} / \mathrm{La} 3 z^{2}-r^{2}$, and Nd/La $x y$ (see SM). Inspecting this orbital, we find that starting from the central $\mathrm{Ni}$ atom, Ni $3 z^{2}-r^{2}$, which bonds to Ni $s$, and antibonds to $\mathrm{O} p_{x} / p_{y}$, bonds strongly with the predominant feature of $\mathrm{Nd} / \mathrm{La} 3 z^{2}-r^{2}$ and $x y$, highlighting the hybridization between $\mathrm{Ni}$ and $\mathrm{Nd} / \mathrm{La}$ $d$. Thus an effective axial orbital is created in a downfolded basis which takes into account all the contributions, other than $\mathrm{Ni} x^{2}-y^{2}$, including that of the interstitial weight. We find that the $\mathrm{Ni} 3 z^{2}-r^{2} / \mathrm{Ni} s$ character is more in the La compound [Fig. 1(f)] compared to the Nd compound [Fig. 1(e)], while the $\mathrm{Nd} 3 z^{2}-r^{2} / \mathrm{Nd} x y$ character is less in the La compound compared to the $\mathrm{Nd}$ compound. This makes the axial orbital much more cylindrical in NNO [Fig. 1(e)] and more spherical in LNO [Fig. 1(f)]. This differential nature of the axial orbital is reflected in both in-plane and out-of-plane hopping interactions within the two-band description (see SM) as well as the energy of the axial orbital $\left(\epsilon_{s}\right)$ measured from the energy of $x^{2}-y^{2}\left(\epsilon_{d}\right)$ lying $0.25 \mathrm{eV}$ higher in NNO compared to that in LNO. The in-plane hopping is found to be $30 \%-20 \%$ larger in NNO compared to LNO. The out-of-plane hopping, especially the hopping connecting axial orbital to axial orbital, shows significantly larger values for NNO compared to LNO (1.2-7 times), accounting for about a $1 \mathrm{eV}$ larger bandwidth of the axial band in NNO compared to LNO.

Calculation of superconducting properties. In analogy with cuprates [37], pnictides [38], and heavy-fermion superconductors [39], we assume superconductivity in the present compound is spin-fluctuation mediated. The estimated electron-phonon interaction turns out to be too small to support the observed $T_{c}$ [17]. Based on a two-band Hubbard model, we obtain the pairing potential by considering the bubble and ladder diagrams [37-40],

$$
\tilde{\Gamma}(\mathbf{q})=\frac{1}{2}\left[3 \tilde{U}_{s} \tilde{\chi}_{s}(\mathbf{q}) \tilde{U}_{s}-\tilde{U}_{c} \tilde{\chi}_{c}(\mathbf{q}) \tilde{U}_{c}+\tilde{U}_{s}+\tilde{U}_{c}\right] .
$$

The "tilde" denotes a tensor in the orbital basis. The subscripts $s$ and $c$ denote spin and charge fluctuation channels, respectively. $\tilde{U}_{s / c}$ are the on-site interaction tensors for spin and charge fluctuations, respectively, whose nonvanishing components are $\left(\tilde{U}_{s, c}\right)_{\alpha \alpha}^{\alpha \alpha}=U_{d / s}$ for intraorbital $x^{2}-y^{2}$ and axial, and the interorbital component is $\left(\tilde{U}_{s, c}\right)_{\alpha \alpha}^{\beta \beta}=V_{s d}(\alpha \neq \beta$ are orbital indices) [41]. $\tilde{\chi}_{s / c}$ are the spin and charge densitydensity correlation functions (tensors in the same orbital basis), computed within the random-phase approximation (see $\mathrm{SM}$ ). The application of a weak-coupling theory may be justified by the fact that the exchange scale in nickelates is smaller than in cuprates.

We compute the eigenvalue and eigenfunctions of the pairing interaction $\tilde{\Gamma}\left(\mathbf{k}-\mathbf{k}^{\prime}\right)$ on the 3D Fermi momenta, by solving the following equation,

$$
\Delta_{v}(\mathbf{k})=-\lambda \frac{1}{\Omega_{\mathrm{BZ}}} \sum_{v^{\prime}, \mathbf{k}^{\prime}} \Gamma_{\nu v^{\prime}}^{\prime}\left(\mathbf{k}-\mathbf{k}^{\prime}\right) \Delta_{v^{\prime}}\left(\mathbf{k}^{\prime}\right) .
$$

Here, $v, v^{\prime}$ denote band indices, and $\Gamma_{v v^{\prime}}^{\prime}$ is the pairing interaction, projected onto the band basis. $\lambda$ is the pairing eigenvalue (proportional to the SC coupling strength), and $\Delta_{v}(\mathbf{k})$ is the corresponding pairing eigenfunction. Since the pairing potential is repulsive here, the highest positive eigenvalue $\lambda$, and the corresponding pairing symmetry, can be shown to govern the lowest free-energy value in the SC state [40].

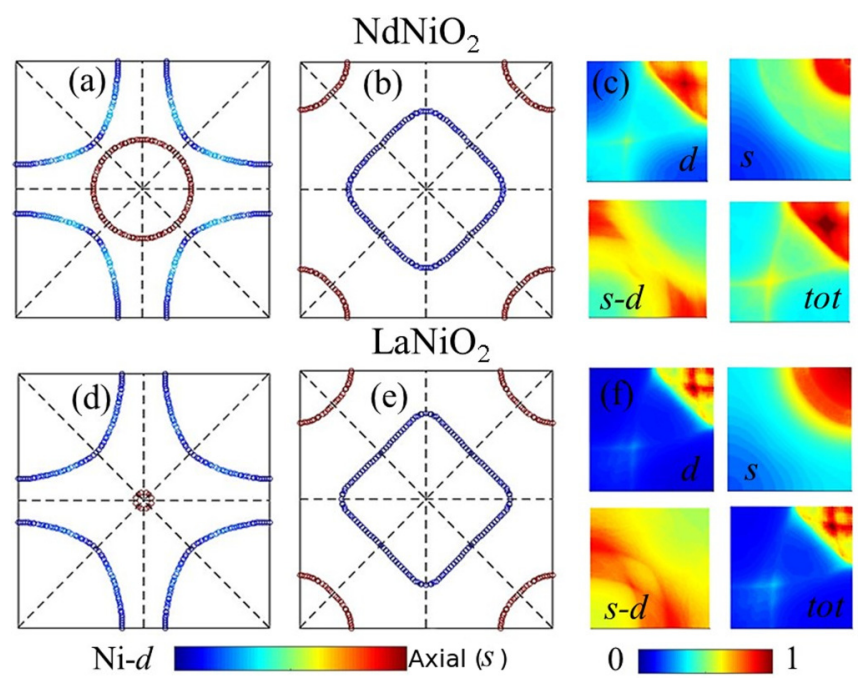

FIG. 2. (a), (b) FS topologies in NNO plotted as a function of $k_{x}-k_{y}\left(-\pi \rightarrow \pi\right.$ range) at $k_{z}=0$, and $\pi$. Blue (Ni $\left.x^{2}-y^{2}\right)$ to red (axial) colors depict the orbital contributions at each $k_{F}$. (c) Plots of static spin susceptibility for two intraorbital, interorbital, and total ( $\operatorname{Tr} \tilde{\chi}_{s}$, summing over both intra- and interorbital contributions) channels, for $q_{z}=\pi$, and $q_{x}, q_{y}: 0 \rightarrow \pi$. All color bars are separately normalized for visualization. (d)-(f) Same as in (a)-(c) but plotted for LNO.

The origin of the unconventional pairing symmetry, and the role of Fermi-surface (FS) nesting can be understood as follows. For $\Gamma>0$ and $\lambda>0$ in Eq. (2), the pairing symmetry $\Delta_{v}(\mathbf{k})$ must change sign over the FS to compensate for the negative sign in the left-hand side of Eq. (2). $\Delta_{v}(\mathbf{k})$ changes sign between the two $\mathbf{q}=\mathbf{k}-\mathbf{k}^{\prime}$ points, and either between different or the same bands which are connected by the momentum $\mathbf{q}$ at which $\Gamma_{v v^{\prime}}^{\prime}(\mathbf{q})$ acquires strong peaks. The loci of the peaks in $\Gamma_{v v^{\prime}}^{\prime}(\mathbf{q})$ are primarily dictated by the FS nesting, while the overall amplitude is determined by $U_{s, d}$ and $V_{s d}$. We fix the hole doping level at $x=0.2$, which is about the optimal doping for NNO [10,28].

In Fig. 2, we show the FS topology for NNO [Figs. 2(a) and 2(b)] and LNO [Figs. 2(d) and 2(e)] at two $k_{z}$ cuts, with the corresponding orbital weight indicated by the red to blue color map. The FSs are seen to be strongly 3D, which is typically detrimental for FS nesting strength. However, owing to the particular orbital weight distributions, there arise dominant nesting channels, which are highly orbital resolved. Interestingly, there is a complete orbital inversion among two FS sheets between $k_{z}=0$ and $\pi$. While the large hole pocket centering the zone boundary and the electron pocket in the zone center of the NNO Brillouin zone (BZ) is of $\mathrm{Ni} d\left(x^{2}-y^{2}\right)$ and axial character $(s)$, respectively, in $k_{z}=0$, they reverse their roles in $k_{z}=\pi$. The Ni $d$ orbital enjoys a FS topology similar to the cuprates case in the low $k_{z}$ region, giving a nearly $2 \mathrm{D}$ FS nesting feature around $\mathbf{Q}=(\pi, \pi, 0)$ and hence a $d_{x^{2}-y^{2}}$-pairing symmetry. On the other hand, the axial orbital acquires FS nesting, considerably weaker in strength compared to the Ni $d$ orbital case, at $\mathbf{Q}=(\pi, \pi, \pi)$, which is responsible for the $3 z^{2}-r^{2}$-type pairing symmetry. The FS for LNO, shown in Figs. 2(d) and 2(e), is topologically similar 


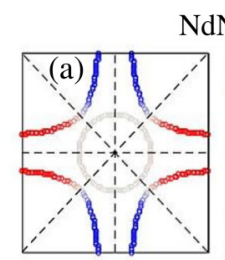

$\mathrm{NdNiO}_{2}$
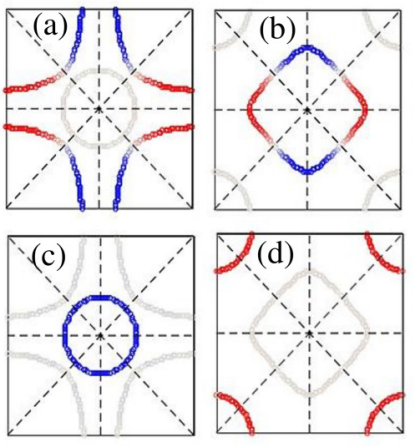

$-1$

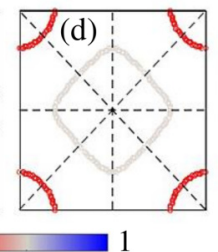

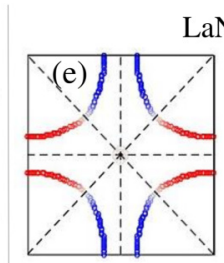

$\mathrm{LaNiO}_{2}$

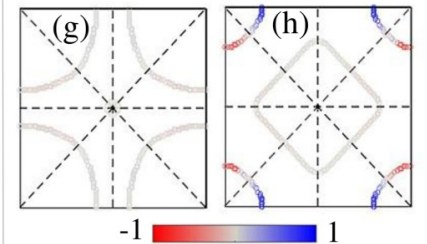

FIG. 3. Computed values of orbital resolved pairing eigenfunction $\Delta_{\alpha}(\mathbf{k})$ plotted on the FS at two representative cuts $k_{z}=0$ [(a)-(c)], $\pi$ [(b)-(d)] for NNO. (a), (b) and (c), (d) give orbital contributions for Ni $x^{2}-y^{2}$ and the axial orbital, respectively. (e)-(h) Same as (a)-(d), but plotted for LNO.

to NNO, except it almost loses its FS pocket at the $\Gamma$ point. Since this heavily weakened FS pocket is dominated by the axial orbital in NNO, the multiband picture is less prominent in LNO. This is also reflected in the far weaker contribution of the interorbital susceptibility, to be discussed in the following.

The orbital-resolved spin susceptibility for NNO is shown in Fig. 2(c), which highlights the importance of the interorbital contribution. The relative contributions from the axial orbital $(s)$ and interorbital $(s-d)$, compared to $\mathrm{Ni} d$, are found to be $1 / 10$ th and $1 / 5$ th, respectively. In comparison, in LNO, they are $1 / 100$ th and $1 / 20$ th, respectively. This makes the total susceptibility dominated almost entirely by the $d$-orbital contribution for LNO, while the significant interorbital orbital contribution makes the total susceptibility in NNO appreciably different from the $d$-orbital contribution [cf. Figs. 2(c) and 2(f)].

In Fig. 3 we plot the pairing eigenfunction $\Delta(\mathbf{k})$ for the highest eigenvalue $\lambda$, but projected onto the different orbital channels as $\Delta_{\alpha \beta}=\sum_{\nu} \Delta_{\nu} \phi_{\nu}^{\alpha *} \phi_{\nu}^{\beta}$ (k dependence is suppressed for simplicity), where $\alpha, \beta$ are orbital indices, and $v$ is the band index. $\phi_{v}^{\alpha}$ is the eigenvector of the two-band Hamiltonian. In NNO, we clearly observe that the pairing symmetry of the Ni $d$ orbital onto the FS is a pure $d_{x^{2}-y^{2}}=$ $\cos k_{x}-\cos k_{y}$ type, with very little or no three-dimensional component. On the other hand, the projected pairing symmetry on the axial orbital can be described by a simple $k_{z}$ dispersion as $\cos k_{z}$, with no signature of the basal plane anisotropy. In contrast, in LNO compound, the axial orbital's contribution to the FS is drastically reduced, and hence the calculated pairing symmetry changes to a simple $d_{x^{2}-y^{2}}$.

Finally, we study how the pairing strength $\lambda$ depends on the choice of the Hubbard interaction parameters, $U_{s}, U_{d}$, and $V_{s d}$, which unravels as an interesting scenario. First, focusing on $\mathrm{NNO}$, we find that $\lambda$ increases almost exponentially with $V_{s d}$ (cf. Fig. 4), while neither $U_{d}$ nor $U_{s}$ is effective in enhancing $\lambda$ [42]. Thus, an appreciable $\lambda$ is obtained only when $V_{s d}$ becomes appreciable. Second, relative to NNO, the pairing

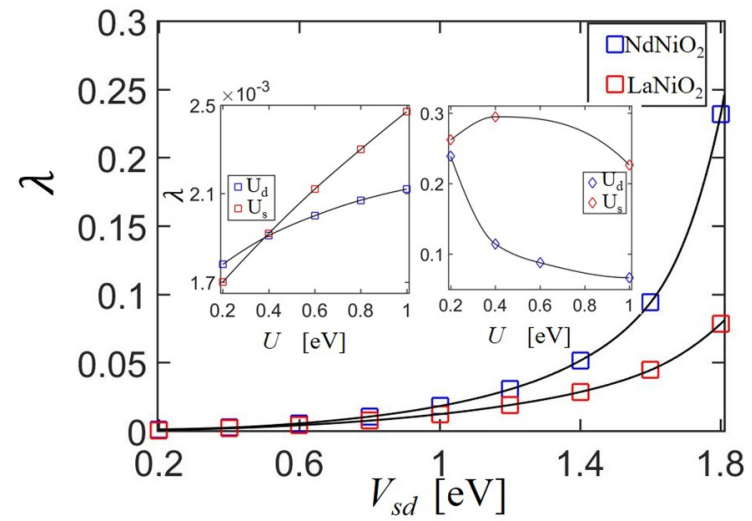

FIG. 4. Evolution of SC coupling constant $\lambda$ as a function of interorbital Hubbard interaction $V_{s d}$ for a choice of $U_{d}=0.9 \mathrm{eV}$ and $0.6 \mathrm{eV}$ for NNO and LNO. The insets show the variation of $\lambda$ as a function of $U_{s}$ and $U_{d}$ in NNO for $V_{s d}=1.5 \mathrm{eV}$ (left) and $V_{s d}=0.5 \mathrm{eV}$ (right) [42].

strength grows much more slowly with $V_{s d}$ in LNO. Thus even for an appreciable value of $V_{s d}$, the pairing strength in LNO is much smaller than NNO. This in turn highlights the important role of the interorbital interaction $V_{s d}$ for superconductivity in nickelate compounds under discussion, and their material dependence.

Conclusion. In summary, motivated by the two-band scenario [22,25,28] proposed for $R \mathrm{NiO}_{2}(R=\mathrm{La}, \mathrm{Nd})$, we derived a two-band Hamiltonian out of DFT calculations, keeping the Ni $x^{2}-y^{2}$ and $\mathrm{Ni} s$ degrees of freedom active, and integrating the rest. The latter forms an axial orbital from a combination of $\mathrm{Nd} / \mathrm{La} d$, Ni $3 z^{2}-r^{2}$, and $\mathrm{Ni} s$, and encodes the material dependence. The calculation of the superconducting properties in such a two-orbital picture shows an orbital-selective pairing for the Nd compound, while it is found to be only of $x^{2}-y^{2}$ symmetry in the La compound. Most importantly, we find that while the SC pairing grows almost in an exponential fashion with the interorbital Hubbard interaction for the $\mathrm{Nd}$ compound, it is not helped by the choice of intraorbital Hubbard interactions. We note that $V_{s d}$ effectively also includes the Hund's coupling. Due to the combined effects of Hund's physics and charge screenings enhanced by $V_{s d}$ [42], the effects of $U_{s, d}$ are presumably superseded by $V_{s d}$. Though the same holds well for the La compound, the growth of a pair interaction with $V_{s d}$ is much weaker than in the $\mathrm{Nd}$ compound, presumably justifying the fact that superconductivity has been so far observed only for the $\mathrm{Nd}$ compound $[10,28]$.

Note added. Recently, we became aware of a tunneling experiment by $\mathrm{Gu}$ et al. [43] reporting two superconducting gaps.

Acknowledgments. T.S.-D. and I.D. acknowledge financial support from the Department of Science and Technology, India. T.S.-D. and I.D. acknowledge support from the National Science Foundation under Grant No. NSF PHY-1748958 and hospitality from KITP where part of this work was performed. T.D.'s research is supported by the STARS-MHRD research fund (STARS/APR2019/PS/156/FS). 
[1] P. A. Lee, N. Nagaosa, and X.-G. Wen, Rev. Mod. Phys. 78, 17 (2006).

[2] A. S. Disa, D. P. Kumah, A. Malashevich, H. Chen, D. A. Arena, E. D. Specht, S. Ismail-Beigi, F. J. Walker, and C. H. Ahn, Phys. Rev. Lett. 114, 026801 (2015).

[3] P. Hansmann, X. Yang, A. Toschi, G. Khaliullin, O. K. Andersen, and K. Held, Phys. Rev. Lett. 103, 016401 (2009).

[4] M. J. Han, Xin Wang, C. A. Marianetti, and A. J. Millis, Phys. Rev. Lett. 107, 206804 (2011).

[5] V. V. Poltavets, M. Greenblatt, G. H. Fecher, and C. Felser, Phys. Rev. Lett. 102, 046405 (2009); V. V. Poltavets, K. A. Lokshin, S. Dikmen, M. Croft, T. Egami, and M. Greenblatt, J. Am. Chem. Soc. 128, 9050 (2006); Ph. Lacorre, J. Solid State Chem. 97, 495 (1992).

[6] S. Sarkar, I. Dasgupta, M. Greenblatt, and T. Saha-Dasgupta, Phys. Rev. B 84, 180411(R) (2011).

[7] V. Pardo and W. E. Pickett, Phys. Rev. Lett. 105, 266402 (2010); Phys. Rev. B 83, 245128 (2011).

[8] M. A. Hayward, M. A. Green, M. J. Rosseinsky, and J. Sloan, J. Am. Chem. Soc. 121, 8843 (1999).

[9] M. A. Hayward and M. J. Rosseinsky, Solid State Sci. 5, 839 (2003).

[10] D. Li, K. Lee, B. Y. Wang, M. Osada, S. Crossley, H. R. Lee, Y. Cui, Y. Hikita, and H. Y. Hwang, Nature (London) 572, 624 (2019).

[11] H. Sakakibara, H. Usui, K. Suzuki, T. Kotani, H. Aoki, and K. Kuroki, Phys. Rev. Lett. 125, 077003 (2020).

[12] X. Wu, D. Di Sante, T. Schwemmer, W. Hanke, H. Y. Hwang, S. Raghu, and R. Thomale, Phys. Rev. B 101, 060504(R) (2020).

[13] J. Karp, A. S. Botana, M. R. Norman, H. Park, M. Zingl, and A. Millis, Phys. Rev. X 10, 021061 (2020).

[14] M. Kitatani, L. Si, O. Janson, R. Arita, Z. Zhong, and K. Held, npj Quantum Mater. 5, 59 (2020).

[15] P. Jiang, L. Si, Z. Liao, and Z. Zhong, Phys. Rev. B 100, 201106(R) (2019).

[16] Y. Gu, S. Zhu, X. Wang, J. Hu, and H. Chen, Commun. Phys. 3, 84 (2020).

[17] Y. Nomura, M. Hirayama, T. Tadano, Y. Yoshimoto, K. Nakamura, and R. Arita, Phys. Rev. B 100, 205138 (2019).

[18] K.-W. Lee and W. E. Pickett, Phys. Rev. B 70, 165109 (2004).

[19] E. Been, W.-S. Lee, H. Y. Hwang, Y. Cui, J. Zaanen, T. Devereaux, B. Moritz, and C. Jia, arXiv:2002.12300.

[20] J. Chang, J. Zhao, and Y. Ding, arXiv:1911.12731.

[21] G.-M. Zhang, Y.-F. Yang, and F.-C. Zhang, Phys. Rev. B 101, 020501(R) (2020).

[22] Y.-H. Zhang and A. Vishwanath, Phys. Rev. Research 2, 023112 (2020).

[23] I. Leonov, S. L. Skornyakov, and S. Y. Savrasov, Phys. Rev. B 101, 241108(R) (2020).

[24] E. A. Stepanov, L. Peters, I. S. Krivenko, A. I. Lichtenstein, M. I. Katsnelson, and A. N. Rubtsov, npj Quantum Mater. 3, 54 (2018)

[25] M. Hepting, D. Li, C. J. Jia, H. Lu, E. Paris, Y. Tseng, X. Feng, M. Osada, E. Been, Y. Hikita, Y.-D. Chuang, Z. Hussain, K. J. Zhou, A. Nag, M. Garcia-Fernandez, M. Rossi, H. Y. Huang, D. J. Huang, Z. X. Shen, T. Schmitt et al., Nat. Mater. 19, 381 (2020).

[26] G. A. Sawatzky, Nature (London) 572, 592 (2019); M. Jiang, M. Berciu, and G. A. Sawatzky, Phys. Rev. Lett. 124, 207004 (2020).
[27] A. Fujimori, J. Club Condensed Matter Phys. (2019), doi: 10.36471/JCCM_December_2019_02.

[28] D. Li, B. Y. Wang, K. Lee, S. P. Harvey, M. Osada, B. H. Goodge, L. F. Kourkoutis, and H. Y. Hwang, Phys. Rev. Lett. 125, 027001 (2020).

[29] G. Kresse and J. Hafner, Phys. Rev. B 47, 558(R) (1993); G. Kresse and J. Furthmüller, ibid. 54, 11169 (1996).

[30] P. E. Blöchl, Phys. Rev. B 50, 17953 (1994).

[31] J. P. Perdew, K. Burke, and M. Ernzerhof, Phys. Rev. Lett. 77, 3865 (1996).

[32] See Supplemental Material at http://link.aps.org/supplemental/ 10.1103/PhysRevB.102.100501 for DFT computational details, a comparison of DFT and Wannier bands, orbital projected band structure for orbital weights forming an axial orbital, and details of the superconductivity calculation.

[33] A. S. Botana and M. R. Norman, Phys. Rev. X 10, 011024 (2020).

[34] A. A. Mostofi, J. R. Yates, Y.-S. Lee, I. Souza, D. Vanderbilt, and N. Marzari, Comput. Phys. Commun. 178, 685 (2008).

[35] E. Pavarini, I. Dasgupta, T. Saha-Dasgupta, O. Jepsen, and O. K. Andersen, Phys. Rev. Lett. 87, 047003 (2001).

[36] E. Pavarini, I. Dasgupta, T. Saha-Dasgupta, and O. K. Andersen, Phys. Rev. Lett. 124, 109701 (2020).

[37] D. J. Scalapino, E. Loh, Jr., and J. E. Hirsch, Phys. Rev. B 34, 8190(R) (1986); 34, 6420 (1986); J. R. Schrieffer, Theory of Superconductivity (W. A. Benjamin, New York, 1964); J. R. Schrieffer, X. G. Wen, and S. C. Zhang, Phys. Rev. B 39, 11663 (1989); P. Monthoux, A. V. Balatsky, and D. Pines, Phys. Rev. Lett. 67, 3448 (1991); M. Sigrist, and K. Ueda, Rev. Mod. Phys. 63, 239 (1991); D. J. Scalapino, ibid. 84, 1383 (2012); J. C. S. David and D.-H. Lee, Proc. Natl. Acad. Sci. 110, 17623 (2013); T. Das, R. S. Markiewicz, and A. Bansil, Adv. Phys. 63, 151 (2014).

[38] I. I. Mazin, D. J. Singh, M. D. Johannes, and M. H. Du, Phys. Rev. Lett. 101, 057003 (2008); S. Graser, T. A. Maier, P. J. Hirschfeld, and D. J. Scalapino, New J. Phys. 11, 025016 (2009); Z.-J. Yao, J.-X. Li, and Z. D. Wang, ibid. 11, 025009 (2009); T. Das and A. V. Balatsky, Phys. Rev. B 84, 014521 (2011); A. Chubukov, Annu. Rev. Condens. Matter Phys. 3, 57 (2012).

[39] T. Takimoto, T. Hotta, and K. Ueda, Phys. Rev. B 69, 104504 (2004); T. Das, J.-X. Zhu, and M. J. Graf, Sci. Rep. 5, 8632 (2015); H. Ikeda, M.-T. Suzuki, and R. Arita, Phys. Rev. Lett. 114, 147003 (2015); T. Nomoto and H. Ikeda, ibid. 117, 217002 (2016); J. Phys. Soc. Jpn. 86, 023703 (2017).

[40] A. V. Chubukov, D. Pines, and J. Schmalian, in The Physics of Superconductors, edited by K. H. Bennemann and J. B. Ketterson (Springer, Berlin, 2003).

[41] Within a two-orbital model, the interaction between the same orbital has been termed as intraorbital, and that between different orbitals has been termed as interorbital. Since the weight of the axial orbital largely belongs to $\mathrm{La} / \mathrm{Nd} d$, the Hund's coupling has not been separately included, though $V_{s d}$ includes this in the sense of a $U^{\prime}$.

[42] We find $\lambda$ varies weakly with $U_{d}$ and $U_{s}$, though quantitative dependency depends on the choice of $V_{s d}$.

[43] Q. Gu, Y. Li, S. Wan, H. Li, W. Guo, H. Yang, Q. Li, X. Zhu, X. Pan, Y. Nie, H.-H. Wen, arXiv:2006.13123. 